\title{
Functional outcomes following combined zacharin's pelvic floor repair and rectopexy for pelvic prolapse
}

\begin{abstract}
Background: Recurrent or large pelvic prolepses are difficult problems. Satisfactory anatomical correction while preserving function and minimizing further recurrence remains a challenge. This study assessed the functional outcome and patient satisfaction following combined Zacharin's procedure (mesh sacrocolpopexy, levatorplasty) and Rectopexy for large and recurrent apical and posterior compartment prolapse.

Methods: Consecutive patients who underwent Zacharin's procedure and Rectopexy were recruited. Medical records were reviewed. Quality of life (QOL) and functional outcome were assessed using P-QOL and PAC SYM questionnaires.

Result: Fifty eight patients had surgery, with a mean age of 64 (46-79). Mean follow up was 3.8 years. Overall, $84 \%$ of patients were satisfied with the outcome of their surgery. There were $4(7 \%)$ recurrent prolepses. Urinary and bowel symptoms improved significantly post surgery $(p<0.001)$. However, the severity of constipation amongst those with ongoing constipation seemed worse.
\end{abstract}

Conclusion: Combined Zacharin's procedure with Rectopexy offers a durable repair with most patients reporting satisfaction, except for those with ongoing constipation.

Summary: Combined Zacharin's procedure and Rectopexy offers patients with large or recurrent apical and posterior vaginal prolapse a durable repair with high patient satisfaction levels, except for those with ongoing constipation.

Keywords: Large pelvic prolepses, Surgical intervention, Abdominal-sacrocolpopexy, Levatorplasty, Recurrent apical prolapse
Volume 7 Issue 4 - 2017

\author{
Cherry E Koh, I,2 Rebecca Deans,3 Andrew \\ Korda,3,4 Christopher J Young I,5 \\ 'Department of Colorectal Surgery, Royal Prince Alfred \\ Hospital,Australia \\ ${ }^{2}$ Surgical Outcomes Research Centre, Royal Prince Alfred \\ Hospital,Australia \\ ${ }^{3}$ Department of Women and Babies, Royal Prince Alfred \\ Hospital,Australia \\ ${ }^{4}$ Western Sydney University, Australia \\ ${ }^{5}$ Discipline of Surgery, University of Sydney, Australia
}

Correspondence: Christopher J Young, Department of Colorectal Surgery, University of Sydney Royal Prince Alfred Hospital, Missenden Rd, Camperdown 2050, NSW, Australia, Tel 61295197576, Email cyoung@aol.com

Received: November 23, 2016 | Published: August 02, 2017

\section{Introduction}

Vaginal prolapse affects up to $50 \%$ of porous women. ${ }^{1,2}$ It has been estimated that up to $10 \%$ of women undergo some form of surgical intervention for prolapse in their life time and that recurrence necessitates re-operation in $29 \%$ of wome. ${ }^{[3-6}$ Management of patients with recurrent or large prolepses can be particularly challenging. Despite this, few publications have addressed the specific issue of optimal management recurrent or large prolepses. ${ }^{7}$ Different surgical repairs have been advocated depending on institutional and surgeon experience and preference. ${ }^{7}$ The vast number of surgical options available would attest to the fact that no single procedure is completely satisfactory. ${ }^{4,6,8}$ Zacharin's procedure which comprises abdominal-sacrocolpopexy and levatorplasty is a procedure that has been advocated for large or recurrent apical prolepses. ${ }^{910}$ Its low recurrence rates coupled with its ability to maintain vaginal patency and therefore sexual function makes it an attractive surgical option. ${ }^{9}$ Because of generalized tissue laxity, some patients may also have coexisting rectal prolapse. ${ }^{8,11,12}$ It has been our practice to treat patients with combined large or recurrent apical prolapse and rectal prolapse using combined Zacharin's procedure and Rectopexy. To date few studies have evaluated functional outcome following surgical repair of pelvic prolapse. The aim of this study was to determine patient satisfaction and functional outcome following combined Zacharin's pelvic floor repair and Rectopexy for patients with large or recurrent apical as well as posterior compartment prolapse.

\section{Methods}

Consecutive patients who underwent combined Zacharin's procedure and Rectopexy between 2000 and 2004 at a single tertiary referral centre were reviewed. Patients were identified by reviewing hospital records and private room records of a single Urogynaecologist (AK). Records were reviewed to retrieve data on demographics, previous surgery, results of Urodynamic and anorectal physiology, pre and post operative symptoms, operative complications and duration of admission. The main outcome of interest was patient perceived satisfaction with surgery as determined by the question "how satisfied are you with the results of your surgery?" Response was scored using a five item discrete Likert scale (Very satisfied, satisfied, somewhat satisfied, dissatisfied and very dissatisfied). This was prospectively assessed at each routine scheduled follow up. Other outcomes of interest were quality of life measures as determined using P QOL (prolapse quality of life), post-operative urinary, bowel and sexual function using the appropriate modules of P QOL and PAC SYM (patient assessment of constipation symptoms). All questionnaires used had been previously validated and found to be valid, reliable and sensitive to change. ${ }^{13,14}$ Patients were recruited by mail, which included a letter explaining the rationale of the study, a consent form and postal questionnaires. Non-responders were sent a reminder to encourage participation.

\section{Pre-operative assessment}

Prolapse was assessed using POPQ (Pelvic Organ Prolapse Quantification system). ${ }^{15}$ All patients with urinary or bowel symptoms routinely underwent Urodynamic and anorectal physiology. Defecating photography was performed when rectal prolapse was suspected but could not be demonstrated. Patients with suspicious bowel symptoms also underwent colonoscopy to rule out a colorectal neoplasm. 


\section{Surgical technique and post-operative patient care}

Details of the procedure have been previously published. ${ }^{9,10,16}$ however, since its original description, several technical modifications have been introduced and these will be described. The procedure is performed with the patient in Lloyd Davies position using a midline incision. Both ureter were identified at the pelvic brim and tracked distally to the pelvic floor. Patients with an intact uterus underwent a hysterectomy. The rectum was fully mobilized into the inter-sphincter plane anteriorly with dissection of the anovaginal septum to the level of dentate line (Figure 1a). The extended rectal dissection is more than what is required for Rectopexy but we found this useful as it allowed the rectum to be displaced further posteriorly to facilitate subsequent levatorplasty. Using a vaginal approach, a longitudinal incision was then made in the posterior wall of the vagina and large vaginal packing gauze was then placed through this incision and tied firmly to the pubic symphysis to retract the bladder and vagina anteriorly leaving the elevator hiatus clearly displayed (Figure 1b). Through this vaginal opening, heavy vicryl sutures were inserted into either side elevator any by the perineal surgeon using a Zacharin's needle (a $20 \mathrm{~cm}$ tapered needle with an eyelet) taking generous bites of the elevator. The abdominal surgeon played a crucial role in retracting the ureter so as not to incorporate them in the levatorplasty and in guiding the safe passage of the Zacharin needle to avoid inadvertent injury to bowel loops. These vicryl sutures were then held in forceps until 3 to 4 levatorplasty sutures were placed. The defect in the vagina is then closed using a separate continuous 3.0 vicryl suture and levatorplasty completed by tying the vicryl sutures. Mesh sacrocolpopexy and Rectopexy using Marceline mesh was then carried out. The mesh was shaped into a "Y" with one limb secured to sacral promontory. The other two limbs of the mesh were used for an offset wrap around the rectum (Figure 1c). Vaginal vault is secured to the longitudinal limb which lies between the rectum and vagina. All patients received bowel preparation and intra-venous antibiotics. A Jackson-Pratt drain and supra-pubic catheter were used routinely. Supra-pubic catheters were removed when post void residual volumes were less than 50 $\mathrm{ml}$ for three consecutive voids. Depending on stool frequency and volume, fiber or aperients were started to optimize stool volume and consistency so as to improve bowel control. Patients were routinely followed up at 6 weeks, 3 and 12 months post-operatively.

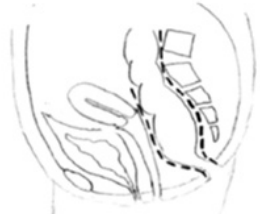

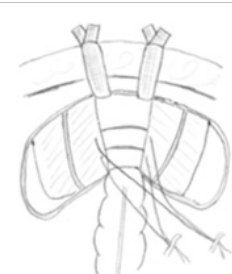

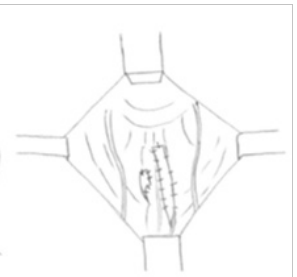

$1 c$
Figure I

a. Sagittal view of the pelvis showing the line of dissection into the intersphincteric plane.

b. Cross section of pelvis showing the levator hiatus held open by two wide ribbon gauzes tied together, both ureter and two levatorplasty sutures held in forceps.

c. View of pelvis showing Rectopexy with offset mesh wrap.

Statistical analyses were performed using SPSS (SPSS Inc, Chicago, Illinois). Non-parametric statistics (McNemar's $\chi 2$ test) were used for comparison of patient satisfaction and to compare QOL between responders and non-responders (Mann Whitney U test). Questionnaires were scored according to the respective scoring manuals and mean scores for PAC SYM were reported. Approval was granted by the local Human Research Ethics Committee.

\section{Results}

Fifty eight women with mean age of 64 (range 46 to 79) had a POPQ Stage IV pelvic organ prolapse. Thirty nine (68\%) patents had endosonography confirmed enterocoeles and the remaining 19 patients had enterocoeles confirmed on defecating photogram. Overt external rectal prolapse was demonstrable in 20 (34\%) patients while the remaining $38(66 \%)$ patients had rectal intussusceptions on defecating photogram. A further $40(69 \%)$ and $33(57 \%)$ patients had concomitant rectocoeles and cystocoeles respectively. Fifty one patients $(88 \%)$ had previously undergone hysterectomy, 45 of which were for prolapse where hysterectomy was part of their previous repair, leaving 13 patients who have not previously had prolapse surgery. Previous prolapse surgery and presenting symptoms are summarized in Table 1 .

Table I Patient previous prolapse surgical history and presenting symptoms

\begin{tabular}{ll}
\hline & $\mathbf{N}(\%)$ \\
\hline Mean Age & 64 \\
Yes & \\
No & $45(78)$ \\
Number of Previous Repairs & $13(22)$ \\
I & \\
2 & $23(40)$ \\
3 & $18(30)$ \\
4 & $3(5)$ \\
Symptoms & $1(2)$ \\
General & \\
Urinary & $58(100)$ \\
Urodynamic Stress Incontinence & $54(93)$ \\
Detrusor Overactivity & $22(41)$ \\
Mixed & $8(15)$ \\
Bowel & $24(44)$ \\
Constipation & $55(93)$ \\
Incontinence & $26(47)$ \\
Mixed Constipation and Incontinence & $12(22)$ \\
\hline
\end{tabular}

In addition to combined Zacharin's procedure and Rectopexy, 7 patients who still had an intact uterus underwent a hysterectomy. 24 underwent simultaneous bilateral salpingo-oophorectomy, 13 patients underwent Burch colposuspension or pubovaginal sling procedure for Urodynamic stress incontinence, 1 underwent left hemicolectomy for colonic polyps and 12 also had incision hernias repaired. There were $9(16 \%)$ minor complications and $2(3 \%)$ major complications within 30 days of surgery. The 9 minor complications were 4 failed trial of voids (all of whom had successful trials of void within 2 weeks post discharge), 2 urinary tract infections and 3 wound infections and superficial wound breakdown. The 2 major complications were pulmonary edema and post-operative arrhythmia. There was no mortality. The average duration of inpatient admission was 9 days (Range 5-30 days). Mean follow-up was 3.8 years (Range 2-5 years). To date, there have been 4 recurrent enterocoeles but none has had mesh erosion into the vagina or rectum.

\section{Patient satisfaction}

At the first post-operative visit, $49(84 \%)$ of patients reported that they were "very satisfied" or "satisfied" with the overall symptomatic relief experienced following surgery (Figure 2). 9 (16\%) patients were "dissatisfied" due to persistent bowel or urinary symptoms despite adequate anatomic correction. None were "very dissatisfied. 
At 12 month follow up, $77 \%$ of patients remained "very satisfied" or "satisfied" with their procedures. Although there was some deterioration in patient satisfaction over the scheduled follow up time period, this was not statistically significant $\left(p=0.125 \mathrm{McNemar} \chi^{2}\right)$. However, there were also 5 patients who were "very dissatisfied". Of the 5 "very dissatisfied" patients, 3 had recurrent enterocoeles, 1 had severe constipation and 1 had ongoing urinary symptoms despite adequate repair. Of the 3 "very dissatisfied" patients with recurrent enterocoeles, 2 have previously had hysterectomy and colposuspension for prolapse and 1 has previously had Zacharin's procedure with silastic ring insertion and were therefore re-recurrences.

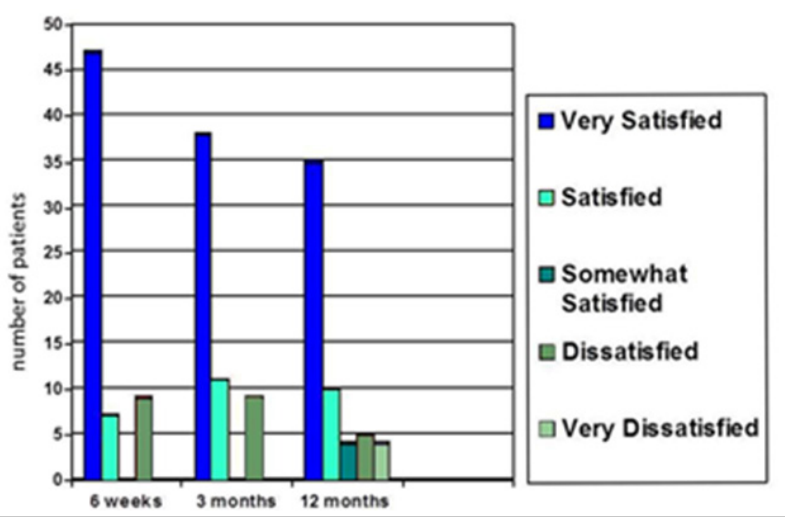

Figure 2 Patient rated satisfaction on a 5 item Likert scale, assessed at each follow up review at 6 weeks, 3 months and 12 months for 58 patients.

\section{Pre and Post-operative symptoms, quality of life assessment}

Pre-operatively, all patients experienced a multitude of symptoms. All complained of non-specific symptoms such as perineal lump, vaginal discomfort and a dull ache. 54 (93\%) patients experienced urinary symptoms and 55 (95\%) had bowel symptoms. Table 1 summarizes the type of urinary and bowel symptoms experienced. Post operative symptoms were assessed based on the $42(72 \%)$ returned questionnaires. Non-responders were sent reminders however no further questionnaires were retrieved. There was no significant difference in satisfaction scores between responders and non-responders to suggest response bias $(\mathrm{p}=0.272$, Mann Whitney $\mathrm{U}$ test). Symptoms were much improved post operatively in all domains including general symptoms of prolapse, urinary as well as bowel symptoms (Table 2). Of the 24 patients who continued to experience urinary symptoms, 10 subsequently underwent urethral sling procedures for Urodynamic stress incontinence with good symptom resolution. In terms of overall quality of life, 3 (7\%), 24 (57\%) and 14 (33\%) participants considered themselves to be in "very good health", "good health" and "fair health" respectively. Only one participant considered themselves to be in "poor health" and none considered themselves to be in "very poor health".

Table 2 Results pre and post-operatively from P QOL questionnaire

\begin{tabular}{llll}
\hline & Pre-op (\%) & Post op (\%) & P\# \\
\hline & $\mathrm{n}=58$ & $\mathrm{n}=42^{*}$ & \\
General Symptoms & $58(100)$ & $9(2 \mathrm{I})$ & $<0.001$ \\
Urinary Symptoms & $54(93)$ & $24(57)$ & $<0.001$ \\
Bowel Symptoms & $55(95)$ & $25(60)$ & $<0.001$ \\
\hline
\end{tabular}

*42 of 58 patients returned questionnaire.

\#Proportion of patients with symptoms pre and post-operatively were compared using Wilcoxon signed rank test.
Of the 38 of $42(90 \%)$ responding patients who continue to experience bowel symptoms, $14(33 \%), 12(29 \%)$ and $12(29 \%)$ had mild, moderate and severe symptoms respectively. Two $(5 \%)$ had transient faucal incontinence while the remaining $36(86 \%)$ complained of some degree of constipation. All patients were referred to biofeedback therapists within the hospital and offered dietary advice, education on the appropriate use of aperients and/or biofeedback. The two patients with faucal incontinence and 20of $36(61 \%)$ patients with any constipation were adequately managed by simple dietary manipulation and/or fiber supplementation. The remaining 16 of $36(44 \%)$ patients reporting constipation $(38 \%$ of questionnaire responders) continued to experience significant constipation despite regular laxatives. All patients subsequently underwent biofeedback therapy and five required colostomies for intractable constipation.

\section{Discussion}

Pelvic floor weakness often affects all three compartments in the perineum, it is therefore not surprising that more than one prolapse can co-exist and for patients to experience urinary and bowel symptoms. ${ }^{8,11}$ In the current series, $91 \%$ and $93 \%$ had concurrent urinary and bowel symptoms respectively. This finding is consistent with reports by other authors. In the series by Lim et al. ${ }^{12} 97 \%$ of their patients experienced urinary or bowel symptoms; in the study by Ellerkman et al. ${ }^{19} 73 \%$ of 237 women were found to have urinary incontinence and $31 \%$ had associated faucal incontinence; in another study by Burrows et al, $59 \%$ and $63 \%$ of 330 patients were found to have stress incontinence and bowel symptoms respectively. ${ }^{18}$ This highlights the importance of assessment of urinary and bowel symptoms prior to attempted surgical repair as targeted uni-compartmental approach of pelvic prolapse are likely to fail or lead to patient dissatisfaction.

The paucity of high quality data in existing literature makes comparing the effectiveness of different types of apical prolapse procedures difficult. Currently, the literature mostly contains evidence derived from retrospective series or uncontrolled studies with few randomized trials. ${ }^{9,16,19} \mathrm{New}$ procedures or modifications are frequently introduced and adopted into practice without concrete scientific backing or useful data. Within these limitations, the surgical approach to the management of apical prolapse is based upon factors such as robustness of repair, recovery time, complications, foreign body risks and the desire for vaginal intercourse. Women and their careers need to evaluate these factors differently and choose a procedure based upon their personal goals for surgery, their general health, and their concern for prolapse recurrence. Overall, cure rates of $79-98 \%$ have been reported in studies with follow up of up to 4 years. ${ }^{19,4}$ With 1014 years follow up, recurrent prolapse occurred in approximately 10 to $25 \%$ of women. ${ }^{20,21}$ In our experience, the Zacharin's procedure compares favorably with these studies. To the best of our knowledge, this is the only case series of Zacharin's procedure published which examines both the short and long term benefits and morbidity following surgery. The procedure is safe with no operative mortality, no mesh erosion and only 2 patient's experienced major morbidity in our cohort of 58 patients. The repair is also durable with only $4(7 \%)$ recurrent enterocoeles and one recurrent rectocoele with almost 4 years follow up. This is highly significant given that $78 \%$ of the patients in this series were recurrent prolepses after initial failed surgical repair and that $38 \%$ of the patients in this cohort have had between one and three additional surgical procedures after their initial hysterectomy for prolapse. Not only is re-operative surgery in this setting challenging, these patients are also likely to have attenuated tissue making durable repair difficult. 
It is well known that prolapse is associated with impaired quality of life. ${ }^{22}$ Yet few studies have evaluated the impact of surgery on quality of life. ${ }^{13}$ Further, the impact of surgery on functional outcome (urinary, bowel and sexual) can be unpredictable and this too is often under reported in existing literature. ${ }^{13}$ A recently updated Cochrane review on the surgical management of prolapse concluded that trials should include patient reported outcomes and that the direct interaction between surgery, bladder, bowel and sexual function needs to be assessed. ${ }^{4}$ In a study by Lim et al assessing the outcome of colpopexy and Rectopexy for prolapse, urinary and bowel symptoms were significantly improved after surgery. In a different study by Milani et al. ${ }^{5}$ improved urinary and bowel function were also reported in small numbers of patients but this was not statistically significant. Although the post-operative improvement in symptoms is statistically significant in this series, it is interesting to note that a significant number of patients continue to experience ongoing symptoms despite adequate anatomical correction of the underlying prolapse and high levels of patient satisfaction. In the study by Milani et al. ${ }^{5}$ despite $94 \%$ of their patients have anatomically satisfactory repairs, $40 \%$ and $30 \%$ continue to experience urinary urgency and constipation. Although Milani et al. ${ }^{5}$ attributed persistent symptoms to the use of proline mesh, it is also known that correlation between symptoms and anatomy is not well defined. ${ }^{2}$ Fitzgerald et al. ${ }^{23}$ found that women with stage II prolapse and previous prolapse surgery reported more symptoms and greater impairment in quality of life compared to those who had more severe prolapse. Conversely, Fritel et al. ${ }^{22}$ found the severity of prolapse was found to be associated with deterioration in quality of life.

One of the limitations of the current study is only post operative quality of life and functional data are available. Further, although the proportion of patients with constipation pre and post surgery is known, the severity of their constipation pre-operatively is not quantified such that we are unable to determine if the severity of constipation was different post operatively. However, anecdotally, it has been our experience that the patients who continue to experience constipation seem to experience more severe constipation. It is known that Rectopexy can be associated with constipation and while it may be tempting to attribute constipation observed in this series to the concomitant Rectopexy, the colorectal surgeons at the same institution have published a ten year series of their Rectopexy results with only $30 \%$ of patients experiencing constipation. ${ }^{24}$ Thus, the inexplicably high rate of constipation observed with this series is above and beyond what would be expected from that from Rectopexy alone. In a paper by Zacharin himself, it was noted that return of bowel function was often "sluggish" although bowel function was thought to be normal after discharge. ${ }^{10}$ A subsequent larger series by Zacharin et al. ${ }^{9}$ similarly did not report on constipation being a problem, however, this may be due to inadequate long term follow up data. In an early series by Korda et al. ${ }^{16} 51$ of 126 women were found to have post-operative constipation. The association between constipation and sacrocolpopexy was recently demonstrated in a study by Forsgren et al. ${ }^{25}$ In their study, women who have previously undergone sacrocolpopexy were found to have higher prevalence of constipation, particularly symptoms of obstructive defecation. ${ }^{25}$ In the current study, 40 of 58 patients also had a concurrent rectocoele that was repaired, which should have improved the mechanics of defecation and hence bowel function, yet this were not the case. Although the mechanism by which this occurs is not clear, in light of this finding, it is important that patients are appropriately informed prior to the procedure.

\section{Conclusion}

Combined mesh sacrocolpopexy, levatorplasty and Rectopexy offers good anatomical correction of apical and rectal prolapse. This repair is safe, durable and associated with good reported patient satisfaction even amongst those with previous failed surgical repairs. However, it is also associated with significant constipation. This has to be balanced against the benefits of the procedure and patients need to be appropriately counseled prior to the procedure.

\section{Conflicts of interest}

The authors do not have any external funding or commercial interest to declare.

\section{Acknowledgments}

The authors are grateful to Dr. Winnie Lau for her assistance in data collection.

\section{References}

1. Beck R, McCormick, L Nordstrom A. 25 year experience with 519 anterior colporrhaphy procedures. Obstet Gynecol. 1991;78(6):10111018 .

2. Digesu G, Chaliha C, Salvatore $\mathrm{S}$, et al. The relationship of vaginal prolapse severity to symptoms and quality of life. Br J Obstet Gynaecol. 2005;112(7):971-976.

3. Thakar R, Stuart Stanton. Management of genital prolapse. BMJ 2002;324(7348):1258-1262.

4. Maher C, Feiner B, Baessler K, et al. Surgical management of pelvic organ prolapse in women. Cochrane Database Syst Rev. 2013;4:CD004014.

5. Milani R, Salvatore S, Soligo M, et al. Functional and anatomical outcome of anterior and posterior vaginal prolapse repair with Proline mesh. BLOG. 2005;112(1):107-111.

6. Weber A, Richter HE. Pelvic Organ Prolapse. Obstet Gynecol. 2005;106:615-634.

7. Gilleran J, Norton PA, Zimmern PE. Vaginal Surgery for Incontinence and Prolapse, Springer-Verlag, London. 2006.

8. Doshani A, Roderick E C Teo, Christopher J Mayne, et al. Uterine prolapse. BMJ. 2007;335:819-823.

9. Zacharin R, NT Hamilton. Pulsion enterocoele: Long term results of an abdominoperineal technique. Obstet Gynecol. 1980;55(2):141-148.

10. Zacharin R, NT Hamilton. The problem of the large enterocoele. Aust NZ J Obstet Gynaecol. 1972;12(2):105-109.

11. Jelovsek J, Maher C, Barber MD. Pelvic organ prolapse. The Lancet. 2007;369:1027.

12. Lim M, Sagar PM, Gonsalves S, et al. Surgical Management of Pelvic Organ Prolapse in Females: Functional Outcome of Mesh Sacrocolpopexy and Rectopexy as a Combined Procedure. Dis Colon Rectum. 2007;50(9):1412-1421.

13. Digesu G, Khullar V, Cardozo L, et al. P-QOL: a validated questionnaire to assess the symptoms and quality of life of women with urogenital prolapse. Int Urogynecol J Pelvic Floor Dysfunc. 2005;16(3):176-181.

14. Frank L, Kleinman L, Farup C, et al. Psychometric validation of a constipation symptom assessment questionnaire. Scand J Gastroenterol. 1999;34(9):870-877.

15. Bump R, Mattiasson A, Bo K, et al. The standardization of female pelvic organ prolapse and pelvic floor dysfunction. Am J Obstet Gynecol. 1996;175(1):10-17. 
16. Kuah S, Lee KW, Houghton CRS, et al. The management of pulsion enterocoele with the Zacharin abdominoperineal technique (and mesh sacrocolpopexy). Aust NZ J Obstet Gynaecol. 2000;40(3):303-307.

17. Ellerkmann RM CG, Melick CF, Nihira MA, et al. Correlation of symptoms with location and severity of pelvic organ prolapse. $\mathrm{Am} \mathrm{J}$ Obstet Gynecol. 2001;185(6):1332-1339.

18. Burrows LJ, Meyn LA, Walters MD, et al. Pelvic Symptoms in Women With Pelvic Organ Prolapse. Obstet Gynecol. 2004;104(5 Pt 1):982-988.

19. Creighton S, Stanton SL. The surgical management of vaginal vault prolapse. Br J Obstet Gynaecol. 1991;98(11):1150-1154.

20. Hilger W, Poulson M, Norton PA. Long term results of abdominal sacrocolpopexy. Am J Obstet Gynecol. 2003;189(6):1606-1610.

21. Aigmuller T, Riss P, Dungl A, et al. Long term follows up after vaginal sacrospinous fixation: patient satisfaction, anatomical results and quality of life. Int Urogynecol J Pelvic Floor Dysfunc. 2008;19(7):965-969.

22. Fritel X, Varnoux N, Zins M, et al. Symptomatic pelvic organ prolapse at midlife, quality of life, and risk factors. Obstet Gynecol. 2009;113(3):609-616.

23. Fitzgerald FP JN, Wren PA, Wei JT, et al. Prolapse severity, symptoms and impact on quality of life among women planning sacrocolpopexy. Int J Gynecol Obstet. 2007;98:24-28.

24. Byrne C, Smith SR, Solomon MJ, et al. Long term functional outcomes after laparoscopic and open rectopexy for the treatment of rectal prolapse. Dis Colon Rectum. 2008;51(11):1597-1604.

25. Forsgren C, Zetterström J, Zhang A, et al. Anal incontinence and bowel dysfunction after sacrocolpopexy for vaginal vault prolapse. Int $J$ Gynecol Obstet. 201021(9):1079-1084. 